\title{
Article
}

\section{Deformation of Surface Nanobubbles Induced by Substrate Hydrophobicity}

\author{
Jiachen Wei, Xianren Zhang, and Fan Song
}

Langmuir, Just Accepted Manuscript • DOI: 10.1021/acs.langmuir.6b03236 • Publication Date (Web): 22 Nov 2016

Downloaded from http://pubs.acs.org on November 29, 2016

\section{Just Accepted}

"Just Accepted" manuscripts have been peer-reviewed and accepted for publication. They are posted online prior to technical editing, formatting for publication and author proofing. The American Chemical Society provides "Just Accepted" as a free service to the research community to expedite the dissemination of scientific material as soon as possible after acceptance. "Just Accepted" manuscripts appear in full in PDF format accompanied by an HTML abstract. "Just Accepted" manuscripts have been fully peer reviewed, but should not be considered the official version of record. They are accessible to all readers and citable by the Digital Object Identifier (DOI®). "Just Accepted" is an optional service offered to authors. Therefore, the "Just Accepted" Web site may not include all articles that will be published in the journal. After a manuscript is technically edited and formatted, it will be removed from the "Just Accepted" Web site and published as an ASAP article. Note that technical editing may introduce minor changes to the manuscript text and/or graphics which could affect content, and all legal disclaimers and ethical guidelines that apply to the journal pertain. ACS cannot be held responsible for errors or consequences arising from the use of information contained in these "Just Accepted" manuscripts. 
Deformation of Surface Nanobubbles Induced by Substrate Hydrophobicity

\author{
Jiachen Wei, ${ }^{\dagger,}$ Xianren Zhang, ${ }^{*, \mathbb{I}}$ and Fan Song*, $\dagger,+$ \\ State Key Laboratory of Nonlinear Mechanics, Institute of Mechanics, Chinese Academy of \\ Sciences, 15 Beisihuanxi Road, Beijing 100190, China, School of Engineering Science, University \\ of Chinese Academy of Sciences, Beijing 100049, China, and State Key Laboratory of \\ Organic-Inorganic Composites, Beijing University of Chemical Technology, Beijing 100029, \\ China \\ E-mail: zhangxr@mail.buct.edu.cn; songf@Inm.imech.ac.cn
}

\begin{abstract}
Recent experimental measurements showed that there exist a population of nanobubbles with different curvature radii, while both computer simulation and theoretical analysis indicated that the curvature radii for different nanobubbles should be the same at a given supersaturation. To resolve such inconsistency, we perform molecular dynamics simulations on surface nanobubbles that are stabilized by heterogeneous substrates in either geometrical heterogeneity model (GHM) or chemical heterogeneity model (CHM), and propose that the inconsistency could be ascribed to the substrate-induced nanobubble deformation. We find that, as expected from theory and computer simulation, for either GHM or CHM there exists a universal upper
\end{abstract}

\footnotetext{
${ }^{*}$ To whom correspondence should be addressed

†'State Key Laboratory of Nonlinear Mechanics, Institute of Mechanics, Chinese Academy of Sciences, 15 Beisihuanxi Road, Beijing 100190, China

${ }^{\ddagger}$ School of Engineering Science, University of Chinese Academy of Sciences, Beijing 100049, China

${ }^{\text {II }}$ State Key Laboratory of Organic-Inorganic Composites, Beijing University of Chemical Technology, Beijing 100029, China 
limit of contact angle for the nanobubbles, which is determined alone by the degree of supersaturation. Through analyzing the shape evolution of nanobubbles as a function of substrate hydrophobicity that is controlled here by the liquid-solid interaction, two different origins of nanobubble deformation are identified. For substrates in GHM that the contact line is pinned by surface roughness, variation in the liquid-solid interaction only changes the location of contact line and the measured contact angle, without causing the change of the nanobubble curvature. For substrates in CHM, however, the liquid-solid interaction exerted by the bottom substrate can deform the vapor-liquid interface, resulting in variation of both the curvature of vapor-liquid interface and the contact angle.

\section{Introduction}

Surface nanobubbles formed on heterogeneous substrates are known for their potential applications in flotation, ${ }^{1}$ boundary slip in fluid, ${ }^{2,3}$ fabrication of bubble-templated nanomaterials ${ }^{4,5}$ and adsorption of macromolecules, ${ }^{6-8}$ all of which entail the initial formation of bubbles with controlled morphology and stability. For instance, stable nanobbubles are desirable to be used as antifouling agents to remove proteins. ${ }^{6}$ Therefore, it is of great interest for generating nanobubbles with controllable shape parameters, including radius of curvature, contact angle and height.

While the formation of nanobubbles is experimentally realized in an oversaturated environmen$\mathrm{t},{ }^{9-11}$ the substrate heterogeneity is crucial for both formation and stability of nanobubbles. ${ }^{9,12,13}$ With numerical simulations, ${ }^{14}$ we have previously demonstrated that surface nanobubbles as well as nanodroplets can be stabilized on a geometrically heterogeneous substrate, on which physical roughness provides the pinning force on nanobubble contact line. ${ }^{15,16}$ Meanwhile, Maheshwari et. al. ${ }^{17}$ have shown that stable surface nanobubble can also form on a chemically heterogeneous substrate, with equilibrium contact angle positively correlated to the supersaturation level of gas molecules. On the other hand, nanobubble collapse could be induced by shock wave, and its damage on solid substrates has also been investigated. ${ }^{18,19}$

The shape of nanobubbles poses a number of challenges in understanding their physical behav- 
iors. $^{20-23}$ In particular, whether neighboring nanobubbles have the same curvature radius or not is still an open question today. Recent experimental measurements revealed that there exist a population of nanobubbles with different curvature radii, ${ }^{24-31}$ while both computer simulations ${ }^{12,14}$ and theoretical analysis ${ }^{9}$ indicated that the curvature radii for different nanobubbles should be the same at a given supersaturation. To resolve this inconsistency, in this work we perform molecular dynamics simulations to show that surface nanobubbles can be stabilized by substrate heterogeneity, either geometrical and chemical, and propose that the inconsistency could be ascribed to the substrate-induced nanobubble deformation.

\section{Simulation method}

\section{Model}

We performed molecular dynamics simulations of surface nanobubbles formed on solid substrates. For all intermolecular interactions, the Lennard-Jones (LJ) $12-6$ interaction was employed. Instead of describing interaction between the particles with the LJ constants $\varepsilon$ and $\sigma$, here we used parameters $A$ and $B$ to separate the LJ potential into repulsive and attractive contributions, which is written as:

$$
U_{i j}=\frac{A_{i j}}{r_{i j}^{12}}-\frac{B_{i j}}{r_{i j}^{6}},
$$

where $A_{i j}=4 \varepsilon \sigma^{12}$ and $B_{i j}=4 \varepsilon \sigma^{6}$ denote respectively the strength of the repulsive and attractive interaction between two particles $i$ and $j$, and $r_{i j}$ is their distance. In our study, $\varepsilon$ and $\sigma$ were chosen as the reduced units for energy and distance, respectively. In the following description we used reduced units, and see Table 1 for the full list of the reduced units.

Quasi-two dimensional box with a size of $73.99 \times 13.15 \times h$ (in unit of $\sigma$ ) was used in this work, as shown in Fig. 1. Periodic boundary conditions were implemented only in $x$ and $y$ directions. In $z$ direction, two restraining substrates that consist of frozen solid molecules in FCC lattice, were placed on the top and bottom of the simulation box. While the bottom substrate was fixed during 
Table 1: Table of reduced units.

\begin{tabular}{|l|l|}
\hline Quantity & Reduced unit \\
\hline Energy & $\varepsilon$ \\
Distance & $\sigma$ \\
A & $4 \varepsilon \sigma^{12}$ \\
$\mathrm{~B}$ & $4 \varepsilon \sigma^{6}$ \\
Temperature & $\varepsilon / k_{\mathrm{B}}$ \\
Pressure & $\varepsilon / \sigma^{3}$ \\
Mass & $M$ \\
Time & $\sqrt{M \sigma^{2} / \varepsilon}$ \\
\hline
\end{tabular}

the simulations, an external force along $z$ direction was uniformly exerted on the top substrate to control the pressure. Therefore, the height of the box, $h$, could fluctuate at a given external pressure $P_{e x}$

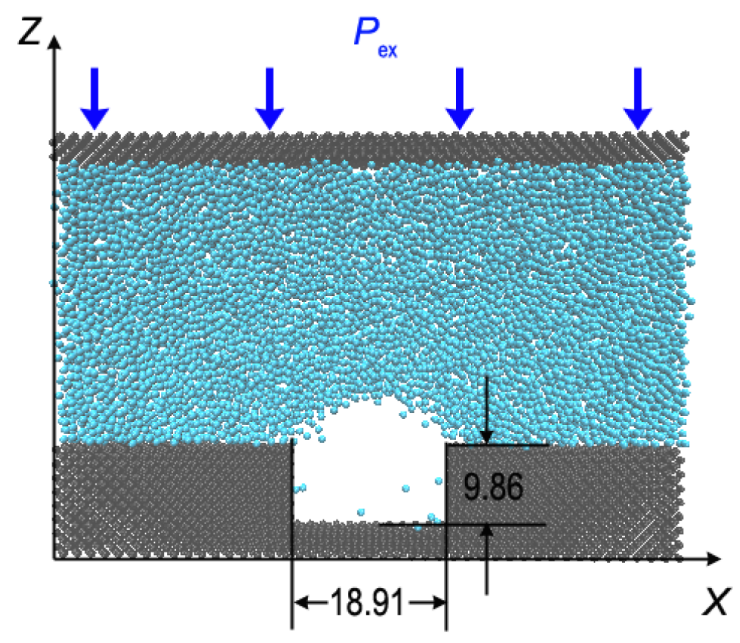

(a)

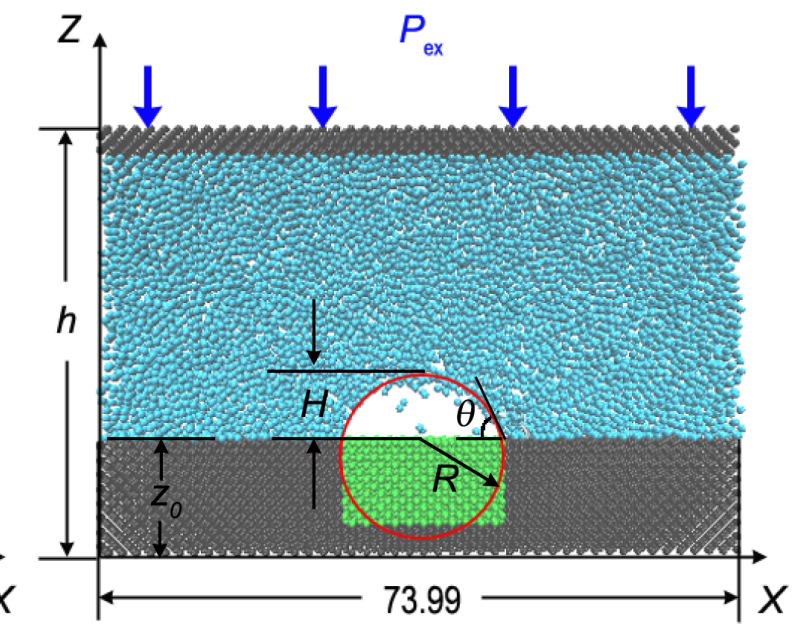

(b)

Figure 1: (a) Geometrical heterogeneity model (GHM). (b) Chemical heterogeneity model (CHM).

Two types of substrate heterogeneity were considered here. For the solid substrates with physical roughness (the geometrical heterogeneity model, GHM), a square pore with a width of 18.91 and a depth of 9.86 was introduced on the substrate to pin the contact line of nanobubbles, see Fig. 1(a). For the solid substrate having chemical heterogeneity (the chemical heterogeneity model, CHM), the square pore was replaced with a cube of more hydrophobic solid that was used to stabilize the nanobubbles, see Fig. 1(b). For both models, the introduced substrate heterogene- 
ity can provide the required pinning force to generate steady-state nanobubbles with uniform and symmetrical interface.

As illustrated in Fig. 1, the vapor-liquid interface of nanobubbles is defined as the locations at which the fluid density is equal to half of the bulk liquid density, which can be fitted by circle approximation:

$$
x^{2}+\left(z-z_{0}+R \cos \theta\right)^{2}=R^{2}
$$

where $z_{0}$ is the height of liquid-solid interface, $R$ the curvature radius and $\theta$ the contact angle. Then, with the obtained vapor-liquid interface, the height of the nanobubble, $H$, is given by

$$
H=R(1-\cos \theta)
$$

\section{Simulation details}

The simulations were performed in $N P_{z z} T$ ensemble, with fixed number of molecules $N=38160$. As mentioned above, an external force along $z$ direction was exerted to maintain the given pressure $P_{e x}$. Such method has frequently been used in the study of the bubble nucleation and proven to be efficient. ${ }^{9,14,32}$ The length of the time step $d t=0.0023$ was carefully chosen, and the Nose-Hoover thermostat with a time constant of $100 d t$ was used to control the temperature of fluids.

All the initial configurations were prepared as follows. First, $N_{f}=23760$ fluid particles were uniformly distributed on FCC crystal lattice between the two substrates to prevent particles from overlapping. The system was then compressed in $z$ direction and quenched to the desired pressure and temperature. Additional long run (at least $8 \times 10^{7}$ simulation steps) was performed to ensure that the system was equilibrated. The data for density distributions were collected by averaging over 1000 output configurations separated by $6 \times 10^{4}$ simulation steps. Unless specified, in this work we fix $T=0.845$ and $P_{e x}=-0.024$. 


\section{Results and discussion}

\section{GHM}

Our simulations show that depending on the substrate hydrophobicity, there exist three different states: vapor, nanobubble, and Wenzel states. The obtained phase diagram for the geometrical heterogeneity model (GHM) in the $A_{l s}-B_{l s}$ plane is presented in Fig. 2(a) for $P_{e x}=0$ and Fig. 2(b) for $P_{e x}=-0.024$. Note that except the liquid-solid interaction parameters, including $A_{l s}$ and $B_{l s}$, all the other parameters for the simulations in the figure kept unchanged, see Table 2. As is shown in Fig. 2(a) and Fig. 2(b), nanobubbles are found to form only when the liquid-solid interactions are moderately hydrophobic. The system would vaporize when the repulsive interaction dominates, and the fluid particles would fill the square pore to form a Wenzel state (i.e., there exists no stable nanobubble) when the attractive interaction becomes sufficiently strong. We also find that as $P_{e x}$ decreases from 0 to -0.024 , the phase boundary for the Bubble-to-Wenzel transition is barely changed.

Table 2: Table of parameters for the Lennard-Jones interaction between different molecules used in GHM.

\begin{tabular}{|l|l|l|}
\hline$i-j$ & $A_{i j}$ & $B_{i j}$ \\
\hline Liquid-liquid $(l-l)$ & 4 & 4 \\
Liquid-solid $(l-s)$ & Var. & Var. \\
Solid-solid $(s-s)$ & 0 & 0 \\
\hline
\end{tabular}

Then, we fix $B_{l s}=4$ and plot the time-averaged density distributions of liquid particles for different values of $A_{l s}$ (Fig. 3). At the rim of pore, the spatial deformation of surface nanobubbles was found due to the interaction between the substrate and the vapor-liquid interface, in a good agreement with AFM measurement by Walczyk et. al. ${ }^{23}$ But far from the substrate, the interface can be well described by spherical fitting of Eq. 2. In contrast to our expectation that the contact line is pinned at a fixed position, we observe that the contact line position shows obvious movement as $A_{l s}$ changes. When $A_{l s}=7$, the attractive force dominates so that the contact line moves downwards and the liquid partially penetrates into the rough substrate. With the increase of the 

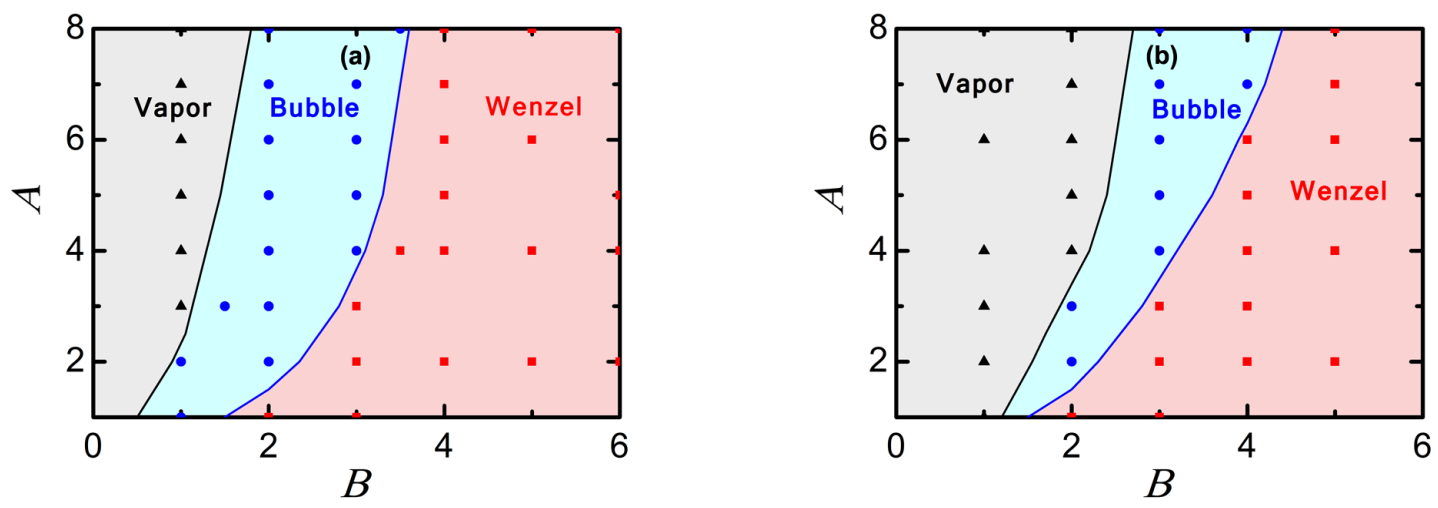

Figure 2: Phase diagram for the state of fluid in $A_{l s}-B_{l s}$ plane with $T=0.845$ for GHM, where (a) $P_{e x}=0$ and (b) $P_{e x}=-0.024$. The red square, blue sphere and black triangle symbols represent the Wenzel, bubble and vapor states, respectively.

repulsion between liquid and solid molecules, the contact line moves upwards along the pore wall until a new force balance is attained. When $A_{l s}=12$, the contact line stops at the mouth of the square pore. Although the curvature radius in this case is almost the same as that for $A_{l s}=7$, the moving of contact line causes the increase of the nanobubble height $H$ and thus increases the measured contact angle $\theta$ according to Eq. 2. When $A_{l s}$ is further increased to 18 , the contact line of the nanobubble even moves slightly across the mouth of the pore (see Fig. 3). Further increasing $A_{l s}$ would induce the depinning of contact line and the nanobubble becomes unstable.
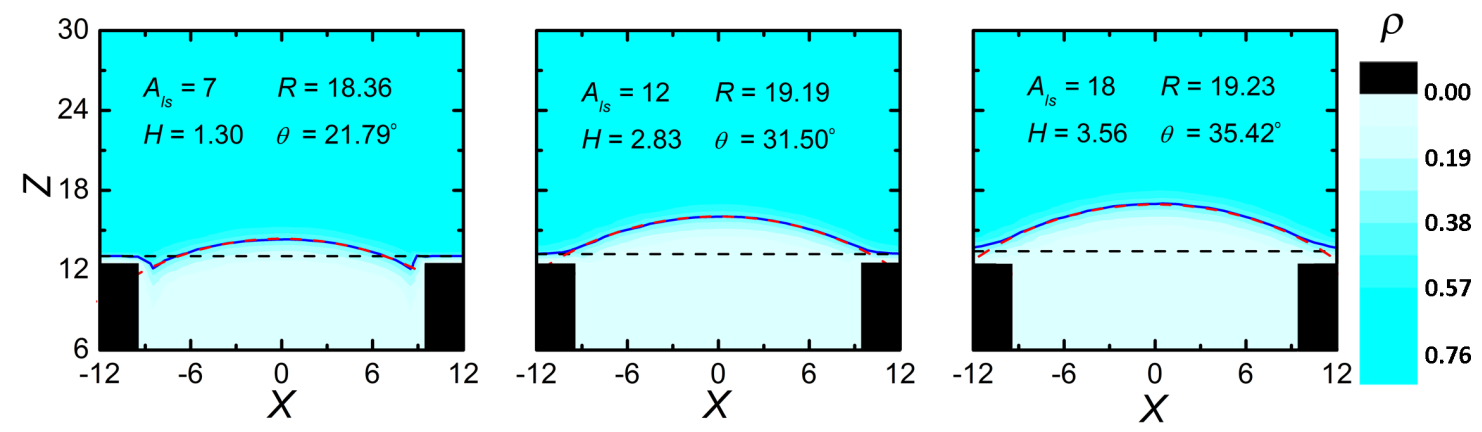

Figure 3: The two-dimensional density distributions of liquid particles for GHM with different values of $A_{l s}$ and fixed $B_{l s}=4$. The blue solid line gives the density contour for $\rho=\rho_{b} / 2$ with $\rho_{b} / 2$ the density of the bulk liquid, which is nearly indistinguishable from the vapor-liquid interface fitted from Eq. 2 (the red dashed line). The black region represents the substrate, and the black dashed line indicates the position of liquid-solid interfaces far from the nanobubble.

For substrates in GHM, the deformation of nanobubbles induced by the variation of liquid- 
solid interaction is illustrated in Fig. 4(a). The most important point is that the contact line shows obvious movement as the fluid-solid interaction changes. With the increase of $A_{l s}$, the vapor-liquid interface moves upwards (from circle $\alpha$ to circle $\gamma$ ), but the curvature radius of the interface almost remains constant. Therefore, neglecting the movement of contact line would induce a deviation of the measured shape parameters of surface nanobubbles from their actual values. Note that the invariance of nanobubble curvature depends on pore width: when pore size is so small that the deformation from opposite contact lines meet, namely the pore size is comparable with the range of liquid-solid interaction, a Cassie state appears instead, ${ }^{14}$ with a curvature radius significantly deviated from that of nanobubbles.

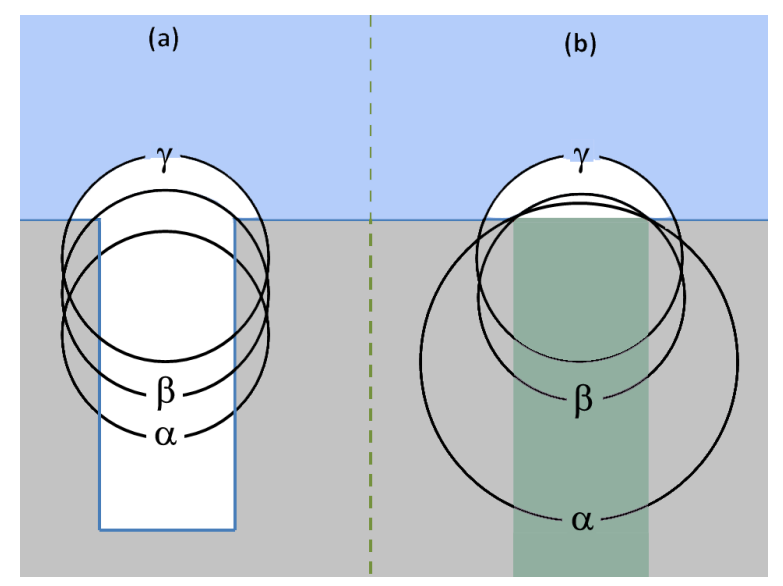

Figure 4: Sketch of nanobubbles deformation due to the increase of repulsion between liquid and solid molecules in fluids for (a) GHM, and (b) CHM.

To further explore the dependence of the bubble shape on liquid-solid intermolecular interactions, Fig. 5(a) and Fig. 5(b) respectively shows the $H-\sqrt{A_{l s}} / B_{l s}$ and $\theta-\sqrt{A_{l s}} / B_{l s}$ relations for different values of $B_{l s}$. Since nanobubbles can appear only within a narrow range of thermodynamic states as shown in Fig. 2(b), we only investigated the relations for $0.6<\sqrt{A_{l s}} / B_{l s}<1.2$. We find that $H$ increases monotonically from 1.30 to 3.85 and $\theta$ from about $21.77^{\circ}$ to $37.24^{\circ}$ with the increase of the repulsive interaction between liquid and solid molecules, and the rate of increase is almost invariant for different $B_{l s}$. Moreover, the upper limits for bubble height $H_{c}=3.85$, and that for contact angle $\theta_{c}=37.24^{\circ}$, depend solely on the external pressure $P_{e x}$, but are independent of $B_{l s}$. 
In general, for nanobubble deformation in GHM, although both $H$ and $\theta$ increase with the increase of the repulsive interaction, the value of curvature radius $R$ is kept unchanged. Moreover, upper limits for bubble height and contact angle are observed, which are, as expected theoretically, 9,12,14 independent of fluid-solid interaction and depend solely on the degree of supersaturation (external pressure in this work).
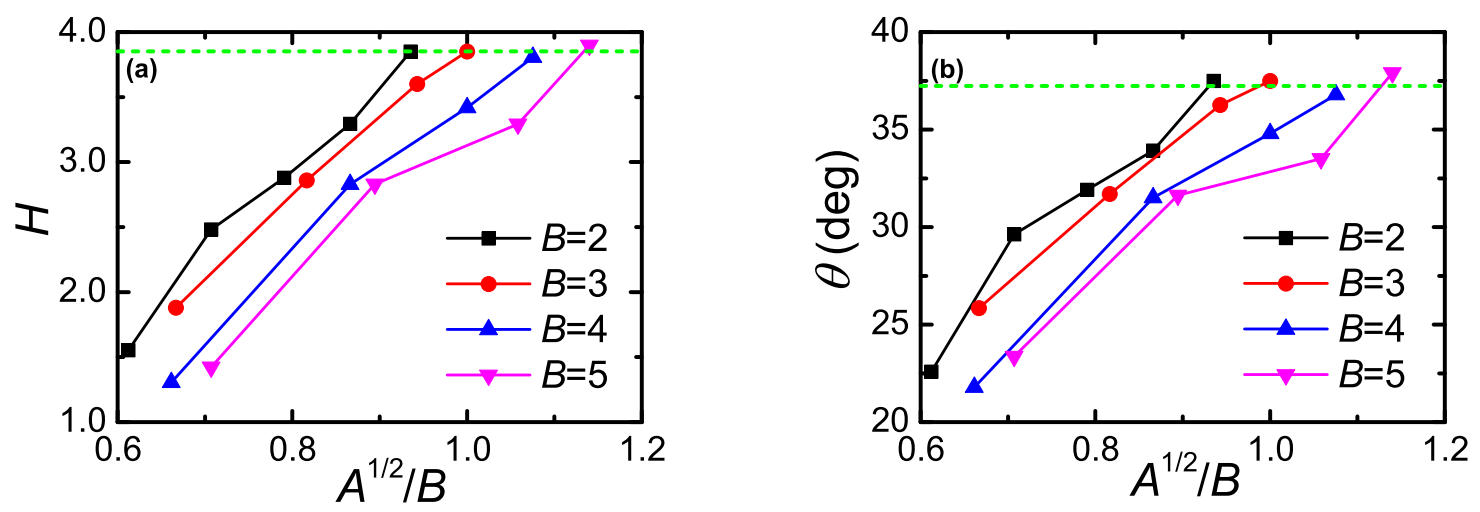

Figure 5: The dependence of (a) nanobubble height $H$, and (b) contact angle $\theta$, on $\sqrt{A} / B$, at different values of $B$ in bubble state for GHM.

\section{CHM}

For the chemical heterogeneity model (CHM) of substrates that are featured with chemical heterogeneity, we fixed $A_{l s}$ and $B_{l s}$, and investigated how the height $H$, the contact angle $\theta$ and curvature radius $R$ depend on the repulsive strength between liquids and the more hydrophobic part of the solid at the bottom of the nanobubble, $A_{l s^{*}}$ (see Fig. 4(b)). The complete set of the parameters for Lennard-Jones interaction used in this subsection are summarized in Table 3.

Table 3: Table of parameters used in CHM.

\begin{tabular}{|l|l|l|}
\hline$i-j$ & $A_{i j}$ & $B_{i j}$ \\
\hline$l-l$ & 4 & 4 \\
$l-s$ & See Fig. 7(a) & See Fig. 7(a) \\
Liquid-hydrophobic solid $\left(l-s^{*}\right)$ & Var. & 1 \\
$s-s$ & 0 & 0 \\
\hline
\end{tabular}


The time-averaged density distributions of liquid particles at $B_{l s^{*}}=1$ and different $A_{l s^{*}}$ are plotted in Fig. 6. When $A_{l s^{*}}=2$, the nanobubble is pinned at the contact line along which solid parts of different hydrophobicity meet. Different from nanobubbles on substrates in GHM, in this case the curvature radius changes significantly as the fluid-solid interaction, $A_{l s^{*}}$, changes. In the case of small $A_{l s^{*}}$ (e.g. $A_{l s^{*}}=2$ ), the contact angle $\theta$ is small and the curvature radius $R$ is relatively large. When $A_{l s^{*}}$ is increased to 4 , the bubble was still pinned at the interface, but we obtain a larger $\theta$ and smaller $R$. However, when $A_{l s^{*}}$ is further increased, for example, to 24 , the liquidsolid contact line of the bubble moves outwards. At this point, while $\theta$ and the nanobubble height $H$ still increases with $A_{l s^{*}}$, the value of $R$ is nearly unchanged. Such tendency of nanobubble deformation is sketched in Fig. 4(b).
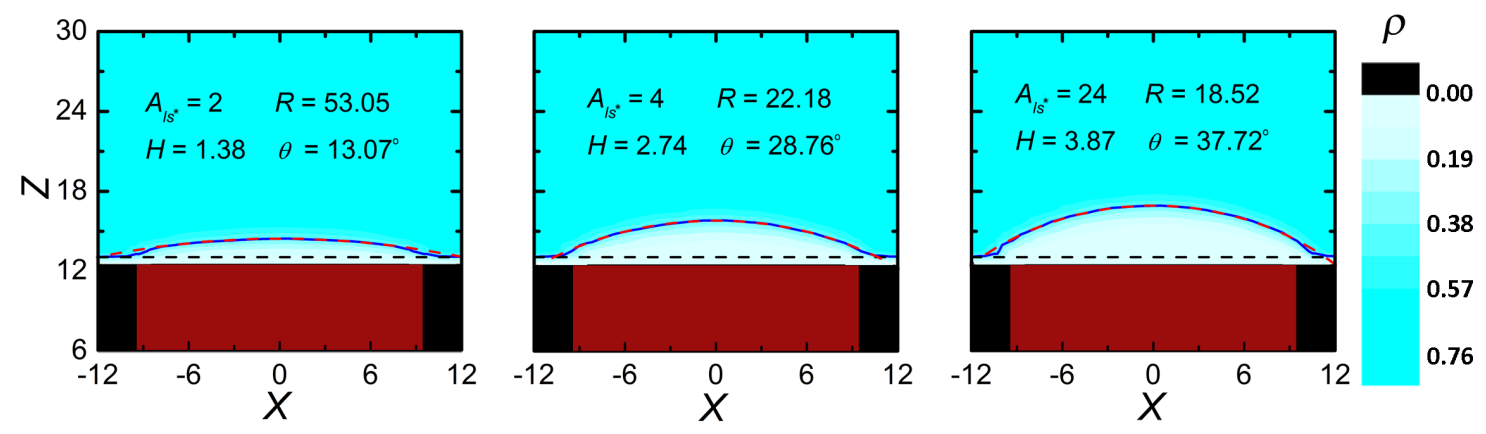

Figure 6: The two-dimensional density distributions of liquid particles for CHM with different liquid-solid interactions. The superscript $s^{*}$ in $A_{l s^{*}}$ denotes the more hydrophobic part of the solid at the bottom (colored in red). Here we fixed $B_{l s^{*}}=1$.

When the nanobubble is pinned at the solid surface, the interaction between the bottom solid surface and the vapor-liquid interface becomes significant at place where their separation distance is small (normally less than $10 \mathrm{~nm}$ ), in particular near the corner of the bubble where the triple phases meet. The varied interaction in reality may arise from nonspecific intermolecular interactions between charged/uncharged particles over the interacting interfaces, which makes the vapor-liquid interface deviate from the ideal state and leads to the change of curvature radius in experiments. This is in accord with our numerical simulations that, by changing the liquid-solid interaction, the curvature of vapor-liquid interfaces changes and the shape of nanobubbles varies.

Fig. 7(a) shows the $R-A_{l s^{*}}$ as well as $\theta-A_{l s^{*}}$ relations for CHM. For any given $A_{l s}$ and 
$B_{l s}$, we observe that, when $A_{l s^{*}}<15$, the increase of the hydrophobicity of the solid substrate below the nanobubbles, i.e., $A_{l s^{*}}$, causes the decrease of $R$ and the increase of $\theta$. When $A_{l s^{*}}>$ 15, however, the values of $R$ and $\theta$ become almost constant. Interestingly, the average values for $A_{l s^{*}}>15$, i.e., $\bar{R}=18.64$ and $\bar{\theta}=37.41^{\circ}$, as illustrated in Fig. 7(a), are almost identical to the corresponding critical values for GHM, that is, $R_{c}=19.22$ and $\theta_{c}=37.24^{\circ}$, at the same thermodynamic conditions. This further confirms that the universal limit of shape parameters for nanobubble is determined alone by the degree of supersaturation ( $P_{e x}$ in this work).
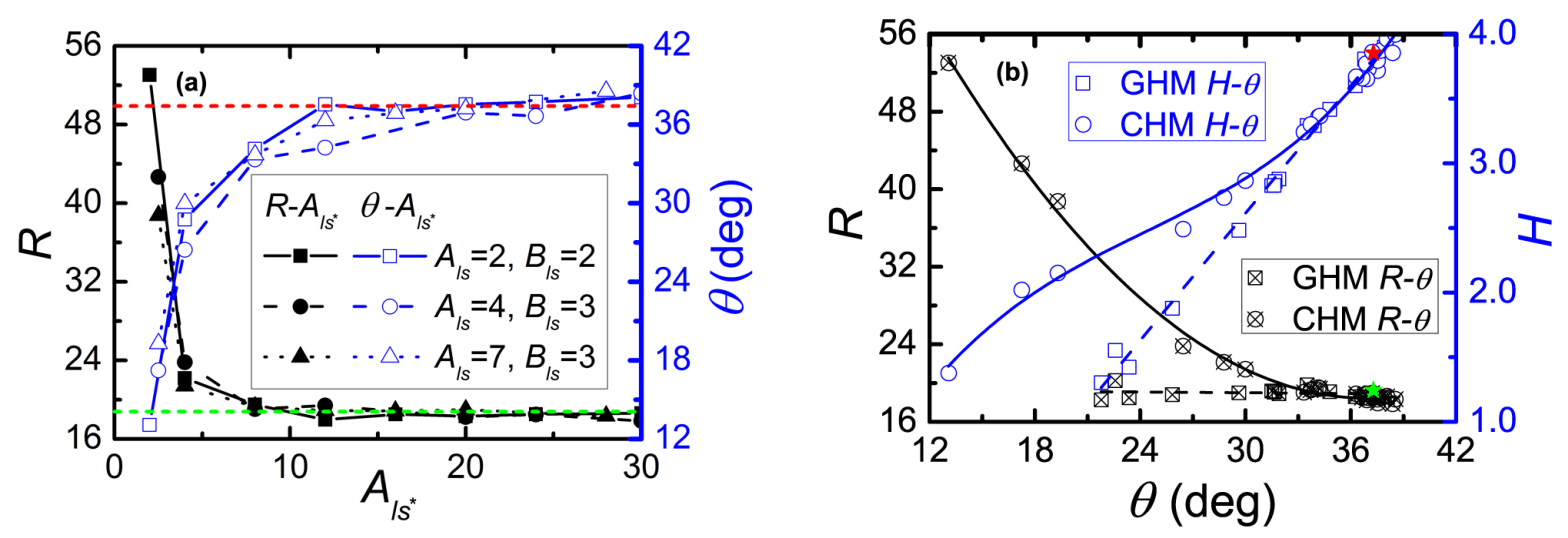

Figure 7: (a) The dependence of curvature radius $R$, and contact angle $\theta$ on $A_{l s^{*}}$, for CHM with fixed $B_{l s^{*}}=1$. The green and red dashed lines give the average value of $\bar{R}=18.64$ and $\bar{\theta}=37.41^{\circ}$ for $A_{l s^{*}}>15$, respectively. (b) The dependence of $R$ and nanobubble height $H$ on $\theta$, for both GHM and CHM with different interaction parameters. The green star represents the expected critical values for curvature radius $R_{c}$ and contact angle $\theta_{c}$, while the red one represents the critical bubble height $H_{c}$.

Such consistency leads us to anticipate that both GHM and CHM may share the same shape of the nanobubbles near the critical point (i.e., without nanobubble deformation). We then give $R$ and $H$ as a function of $\theta$ for both GHM and CHM in Fig. 7(b). We find that nanobubbles have larger $R$ and $H$ in CHM than in GHM at the same $\theta$, especially when $\theta<34^{\circ}$. However, if the liquid-solid repulsive interaction is further increased $\left(\theta>34^{\circ}\right)$, the shape of bubbles for GHM and CHM would converge. Therefore, as expected, the upper limits of $H$ and $\theta$, and lower limit of $R$ for $\mathrm{CHM}$ are quite close to our previous observation of the critical values (i.e., $H_{c}, R_{c}$ and $\theta_{c}$ ) for GHM, as illustrated in Fig. 7(b). 


\section{A heterogeneous model combining GHM and CHM}

In reality the effects from GHM and from CHM may be superimposed. Here, we introduced a heterogeneous substrate model combining both GHM and CHM, in which the hydrophobic patch of the substrate in CHM was placed beside a pore in GHM, to analyze the coupling effect. The complete set of the parameters for LJ interaction used here are summarized in Table 4.

Table 4: Table of parameters used for the combined model.

\begin{tabular}{|l|l|l|}
\hline$i-j$ & $A_{i j}$ & $B_{i j}$ \\
\hline$l-l$ & 4 & 4 \\
$l-s$ & 2 & 2 \\
Liquid-hydrophobic solid $\left(l-s^{*}\right)$ & Var. & 1 \\
$s-s$ & 0 & 0 \\
\hline
\end{tabular}

Fig. 8(a) shows a typical averaged density distribution of liquid particles obtained from the simulations at $A_{l s^{*}}=30$ and $B_{l s^{*}}=1$. Obviously, non-spherical shaped nanobubbles were observed due to the asymmetric nature of the pore characteristics. For comparison, we fit the left and right parts of the vapor-liquid interface with Eq. 2, respectively, and the difference between the simulated interface and the fitted one from the opposite part clearly indicates the non-spherical shape of nanobubbles.
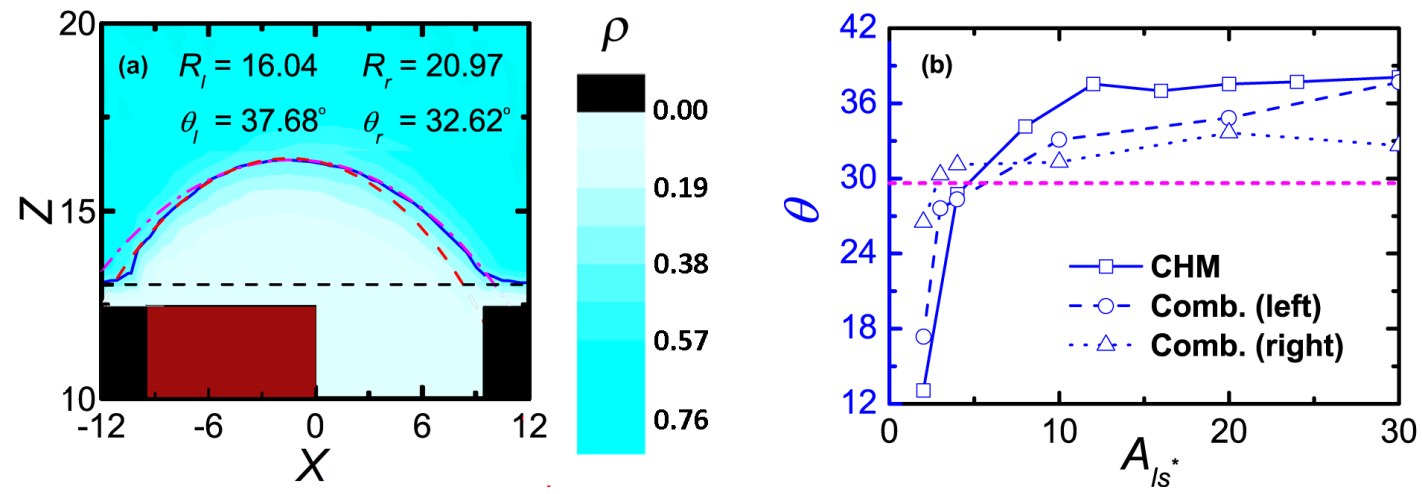

Figure 8: (a) The two-dimensional density distributions of liquid particles for the combined model, with fixed $A_{l s^{*}}=30$ and $B_{l s^{*}}=1$. The red and margarita dashed lines respectively give the spherical fit for the left and right side of the vapor-liquid interface. (b) The dependence of contact angle $\theta$ on $A_{l s^{*}}$ for the combined model, with $B_{l s^{*}}=1$. The $\theta-A_{l s^{*}}$ relation for CHM is also plotted for comparison. The margarita dashed line gives the contact angle of same interaction parameters $\theta_{G H M}=29.62^{\circ}$ for $\mathrm{GHM}$. 
To quantitatively analyze how the shape of nanobubbles deforms when both GHM and CHM are superimposed, the corresponding $\theta-A_{l s^{*}}$ relations from our simulations is shown in Fig. 8(b). The contact angle from the right side fluctuates around $30^{\circ}$, which is very close to that obtained from GHM with the same interaction parameters $\left(\theta_{G H M}=29.62^{\circ}\right)$. The contact angle from the left side of the pore, however, increases significantly with the increase of liquid-solid repulsion for $A_{l s^{*}}<5$, and increases slightly for $A_{l s^{*}}>5$ until reaching the critical contact angle for CHM $\left(\theta_{C H M} \sim 37.41^{\circ}\right)$. In principle, the contact angle from left side is dominated by CHM while that from right side is dominated by the GHM, but the coupling effect of the superimposed substrate would bridge the gap between $\theta_{G H M}$ and $\theta_{C H M}$ by slightly enlarging or reducing $\theta$ of both sides.

\section{Conclusion}

To sum up, we have demonstrated that the curvature radii of nanobubbles can change with the liquid-solid interactions in a wide range. We considered here two models of surface heterogeneity that provides the pinning force for stabilizing nanobubbles: geometrical heterogeneity model (GHM) and chemical heterogeneity model (CHM). Our molecular dynamics simulations demonstrate that the deformation of nanobubbles is induced by substrate hydrophobicity that is controlled by the liquid-substrate interaction. Through analyzing the shape evolution of nanobubbles, two different origins of nanobubble deformation are identified. For the substrate in GHM that the contact line is pinned by surface roughness, variation in the liquid-solid interaction causes the change of the location of contact line, but not the nanobubble curvature, as long as the pore size is sufficiently larger that the range of liquid-solid interaction. For the substrate in CHM, however, the liquid-solid interaction exerted by the bottom substrate would deform the vapor-liquid interface of nanobubbles when the interface is within the range of the interaction, resulting in a curvature deviated from that for nanobubbles free of deformation. Our simulations also show that the height and contact angle of the bubble are positively correlated to the liquid-solid interaction, with the universal upper limits determined both for CHM and GHM. 
Our simulation results can be used to interpret the inconsistency between experimental observations and theoretical investigations: experiments showed that there exist a population of nanobubbles with different curvature radii, while both computer simulation and theoretical analysis indicated that the curvature radii for different nanobubbles should be the same at a given supersaturation. Here we ascribe the inconsistency to the difference of nanobubble deformation caused by geometrical heterogeneity and chemical heterogeneity. Further, since it is experimentally applicable to control the chemical or geometrical distribution of the substrate, our results may prove useful in fabricating nanobubbles for the adsorption of macromolecules and the self-assembly of materials with specific curvature.

\section{Acknowledgement}

This work is supported by National Natural Science Foundation of China (Nos.11602279, 21276007, $11232013,11472285$ and 91434204$)$.

\section{References}

(1) Hampton, M. A.; Nguyen, A. V. Accumulation of dissolved gases at hydrophobic surfaces in water and sodium chloride solutions: Implications for coal flotation. Minerals Engineering 2009, 22, 786-792.

(2) Wang, Y.; Bhushan, B. Boundary slip and nanobubble study in micro/nanofluidics using atomic force microscopy. Soft Matter 2010, 6, 29-66.

(3) Bhushan, B.; Pan, Y.; Daniels, S. AFM characterization of nanobubble formation and slip condition in oxygenated and electrokinetically altered fluids. Journal of Colloid and Interface Science 2013, 392, 105-116.

(4) Hui, F.; Li, B.; He, P.; Hu, J.; Fang, Y. Electrochemical fabrication of nanoporous polypyrrole film on HOPG using nanobubbles as templates. Electrochemistry Communications 2009, 11, $639-642$. 
(5) Lohse, D.; Zhang, X. Surface nanobubbles and nanodroplets. Reviews of Modern Physics 2015, 87,981 .

(6) Wu, Z.; Chen, H.; Dong, Y.; Mao, H.; Sun, J.; Chen, S.; Craig, V. S. J.; Hu, J. Cleaning using nanobubbles: Defouling by electrochemical generation of bubbles. Journal of Colloid and Interface Science 2008, 328, 10-14.

(7) Liu, G.; Wu, Z.; Craig, V. S. J. Cleaning of Protein-Coated Surfaces Using Nanobubbles: An Investigation Using a Quartz Crystal Microbalance. The Journal of Physical Chemistry C 2008, 112, 16748-16753.

(8) Koshiyama, K.; Wada, S. Collapse of a lipid-coated nanobubble and subsequent liposome formation. Nature Scientific Reports 2016, N/A, N/A.

(9) Lohse, D.; Zhang, X. Pinning and gas oversaturation imply stable single surface nanobubbles. Physical Review E 2015, 91, 031003.

(10) Weijs, J. H.; Snoeijer, J. H.; Lohse, D. Formation of Surface Nanobubbles and the Universality of Their Contact Angles: A Molecular Dynamics Approach. Physical Review Letters 2012, 108, 104501.

(11) Weijs, J. H.; Lohse, D. Why Surface Nanobubbles Live for Hours. Physical Review Letters 2013, 110,054501 .

(12) Liu, Y.; Zhang, X. Nanobubble stability induced by contact line pinning. The Journal of Chemical Physics 2013, 138, 014706.

(13) Zhang, X.; Chan, D.; Wang, D.; Maeda, N. Stability of interfacial nanobubbles. Langmuir 2013, 29, 1017-1023.

(14) Liu, Y.; Zhang, X. A unified mechanism for the stability of surface nanobubbles: Contact line pinning and supersaturation. The Journal of Chemical Physics 2014, 141, 134702. 
(15) Liu, Y.; Zhang, X. Evaporation dynamics of nanodroplets and their anomalous stability on rough substrates. Physical Review E 2013, 88, 012404.

(16) Ritchie, J. A.; Yazdi, J. S.; Bratko, D.; Luzar, A. Metastable Sessile Nanodroplets on Nanopatterned Surfaces. The Journal of Physical Chemistry C 2012, 116, 8634-8641.

(17) Maheshwari, S.; Van de Hauf, M.; Zhang, X.; Lohse, D. Stability of Surface NanobubblesA Molecular Dynamics Study. Langmuir 2016, N/A, N/A.

(18) Shekhar, A.; Nomura, K.-i.; Kalia, R. K.; Nakano, A.; Vashishta, P. Nanobubble Collapse on a Silica Surface in Water: Billion-Atom Reactive Molecular Dynamics Simulations. Physical Review Letters 2013, 111, 184503.

(19) Vedadi, M.; Choubey, A.; Nomura, K.; Kalia, R. K.; Nakano, A.; Vashishta, P.; van Duin, A. C. T. Structure and Dynamics of Shock-Induced Nanobubble Collapse in Water. Physical Review Letters 2010, 105.

(20) Guo, Z.; Liu, Y.; Xiao, Q.; Schonherr, H.; Zhang, X. Modeling the interaction between AFM tips and pinned surface nanobubbles. Langmuir 2016, 32, 751-758.

(21) Walczyk, W.; Schonherr, H. Closer look at the effect of AFM imaging conditions on the apparent dimensions of surface nano-bubbles. Langmuir 2013, 29, 620-632.

(22) Walczyk, W.; Schon, P. M.; Schonherr, H. The effect of PeakForce tapping mode AFM imaging on the apparent shape of surface nanobubbles. Journal of physics: Condensed matter 2013, 25, 184005.

(23) Walczyk, W.; Schonherr, H. Dimensions and the proïñ̌le of surface nanobubbles: tipấĽ́nanobubble interactions and nanobubble deformation in atomic force microscopy. Langmuir 2014, 30, 11955-11965.

(24) Zhang, X.; Maeda, N.; Craig, V. S. J. Physical properties of nanobubbles on hydrophobic surface in water and aqueous solutions. Langmuir 2006, 22, 5025-5035. 
(25) Yang, S.; Dammer, S. M.; Bremond, N.; Zandvliet, H. J. W.; Kooij, E. S.; Lohse, D. Characterization of nanobubbles on hydrophobic surfaces in water. Langmuir 2007, 23, 7072-7077.

(26) Zhang, X.; Quinn, A.; Ducker, W. A. Nanobubbles at the interface between water and a hydrophobic solid. Langmuir 2008, 24, 4756-4764.

(27) Yang, S.; Tsai, P.; Kooij, E. S.; Prosperetti, A.; Zandvliet, H. J. W.; Lohse, D. Electrolytically generated nanobubbles on highly orientated pyrolytic graphite surfaces. Langmuir 2009, 25, $1466-1474$.

(28) Brotchie, A.; Zhang, X. Response of interfacial nanobubbles to ultrasound irradiation. Soft Matter 2011, 7, 265-269.

(29) Zhang, X.; Uddin, M. H.; Yang, H.; Toikka, G.; Ducker, W. A.; Maeda, N. Effects of surfactants on the formation and the stability of interfacial nanobubbles. Langmuir 2012, 28, $10471-10477$.

(30) Zhang, X.; Lohse, D. Perspectives on surface nanobubbles. Biomicrofluidics 2014, 8, 041301.

(31) German, S. R.; Wu, X.; An, H.; Craig, V. S. J.; Mega, T. L.; Zhang, X. Interfacial nanobubbles are leaky: Permeability of the gas/water interface. ACS nano 2014, 8, 6193-6201.

(32) Novak, B. R.; Maginn, E. J.; McCready, M. J. Comparison of heterogeneous and homogeneous bubble nucleation using molecular simulations. Physical Review B 2007, 75, 085413. 


\section{Graphical TOC Entry}

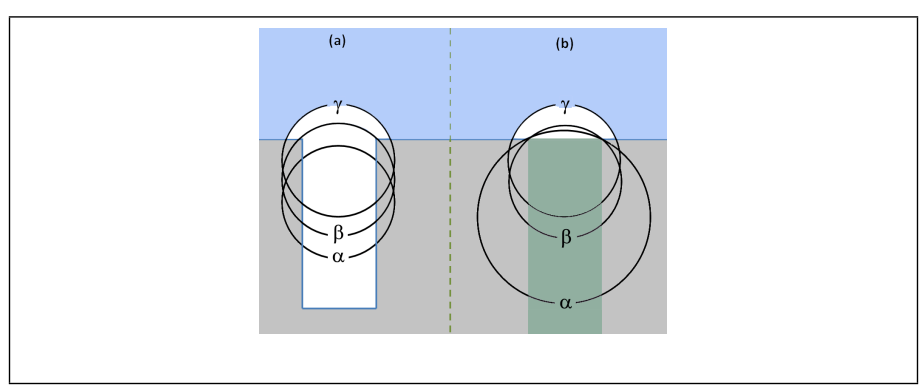

16

17

18

19

20

21

22

23

24

25

26

27

28

29

30

31

32

33

34

35

36

37

38

39

40

41

42

43

44

45

46

47

48

49

50

51

52

53

54

55

56

57

58

59

60 


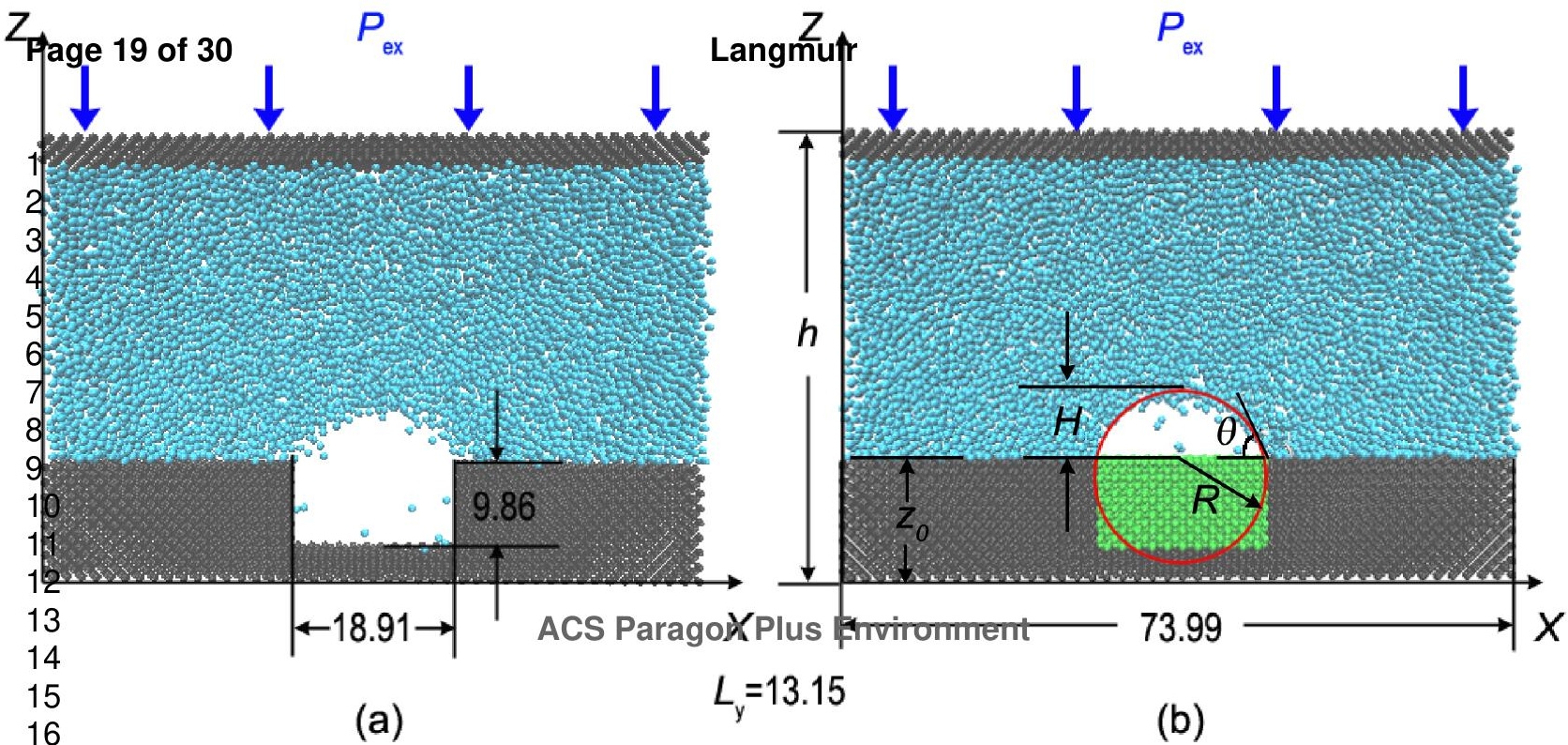




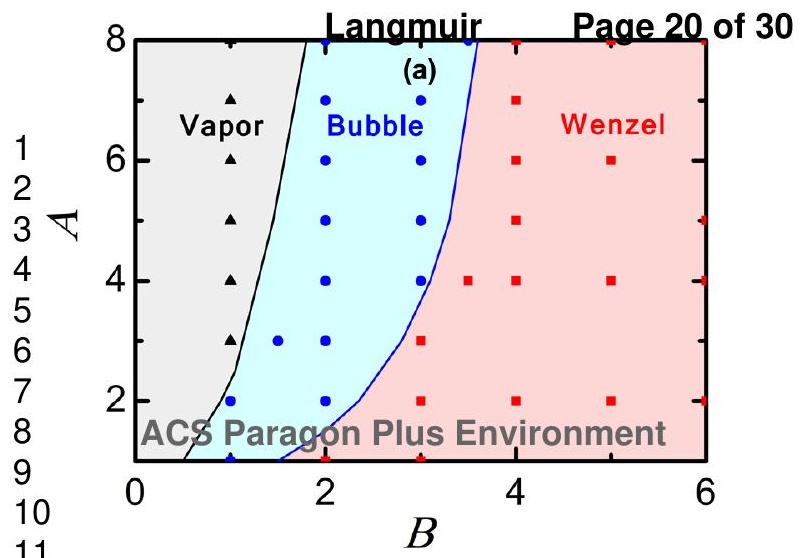




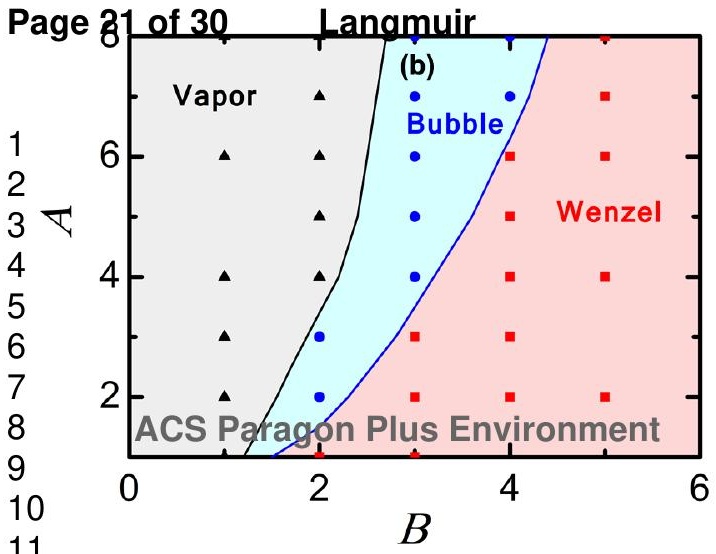


Page 23 of 30

(a)

Langmuir

(b)

1
2
3
4
5
6
7
8
9
10
11
12
13
14
15
16
17
18
19
20
21
22
23
24
25
26
27
28
29
30
31
32
33
34
35
36
38
39
40

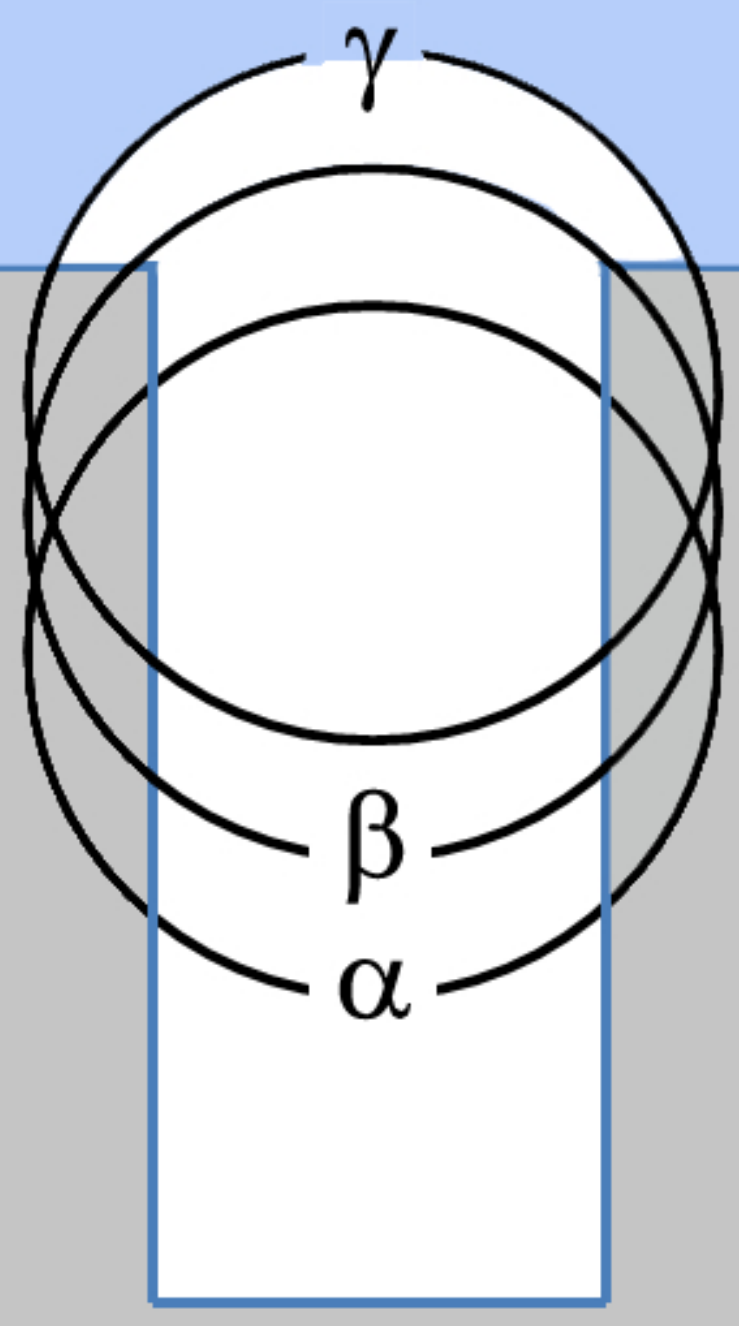

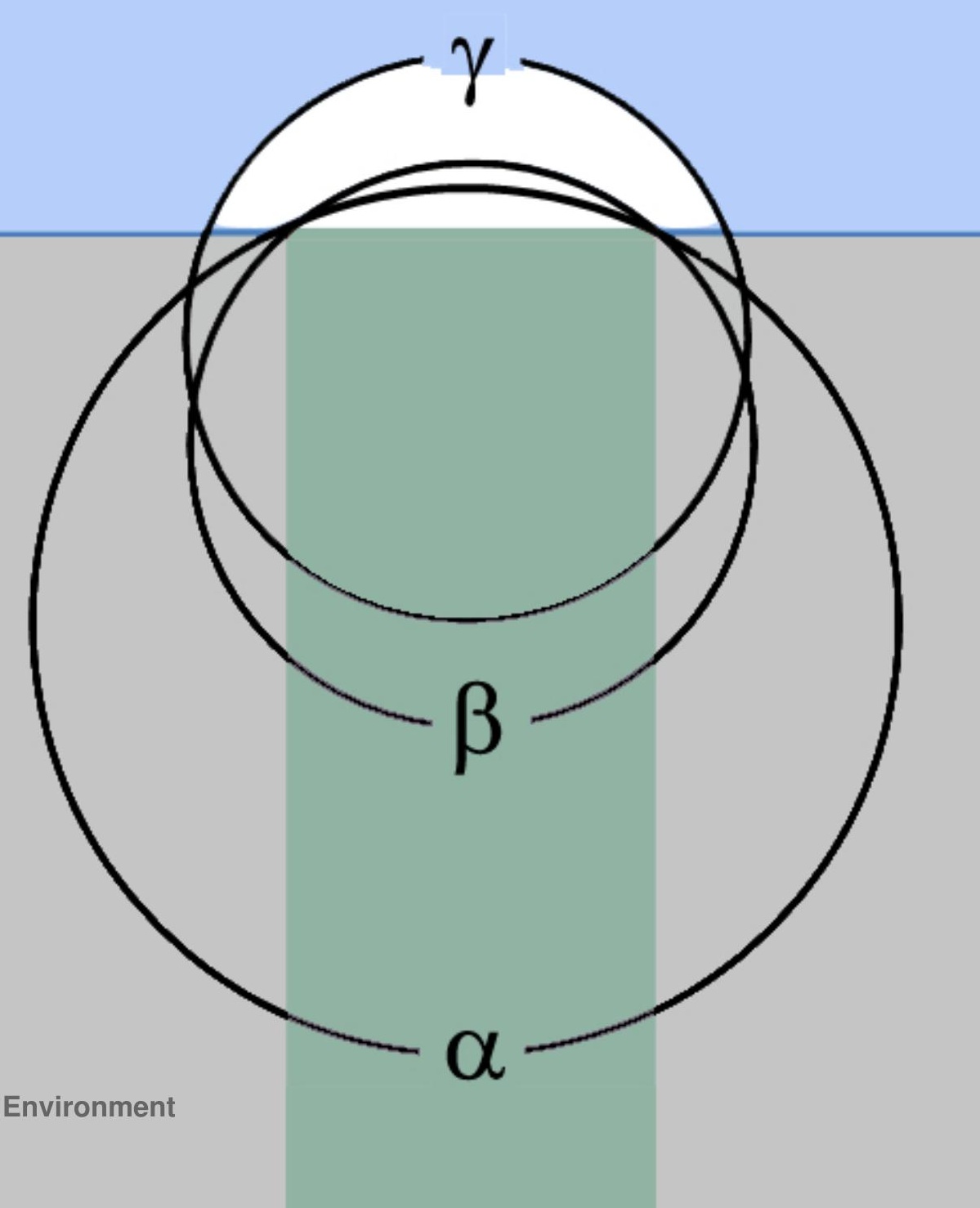




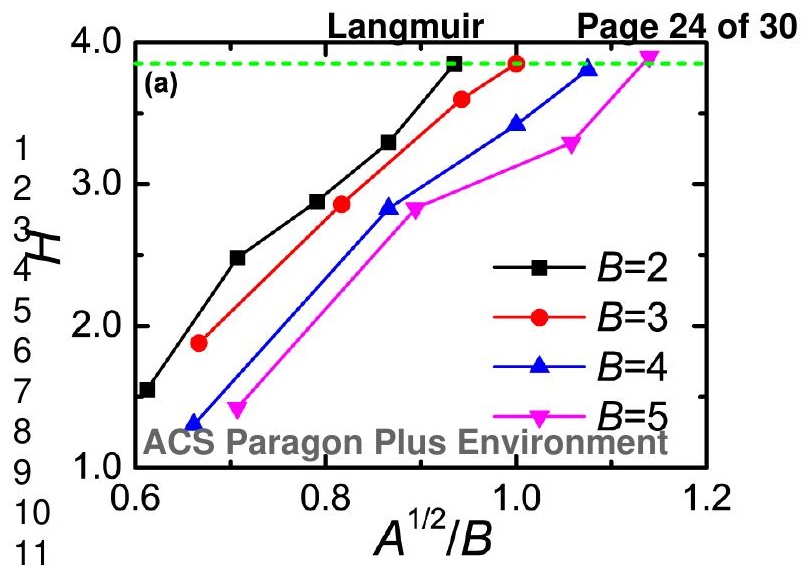


Page 35 of 30 , Langmuir

(b)

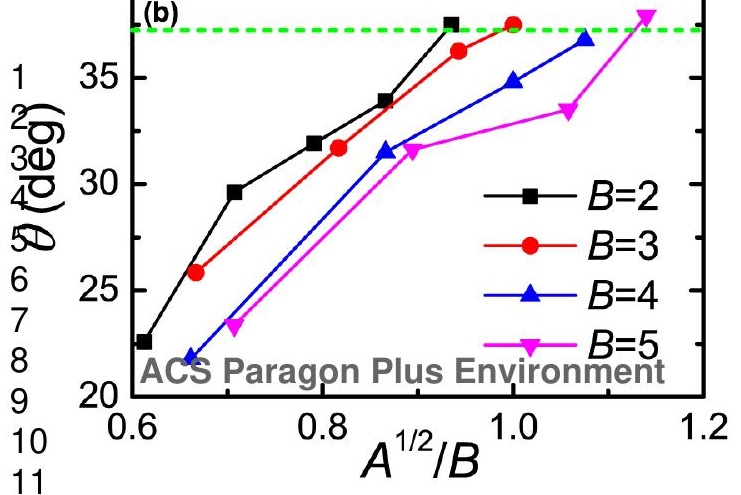




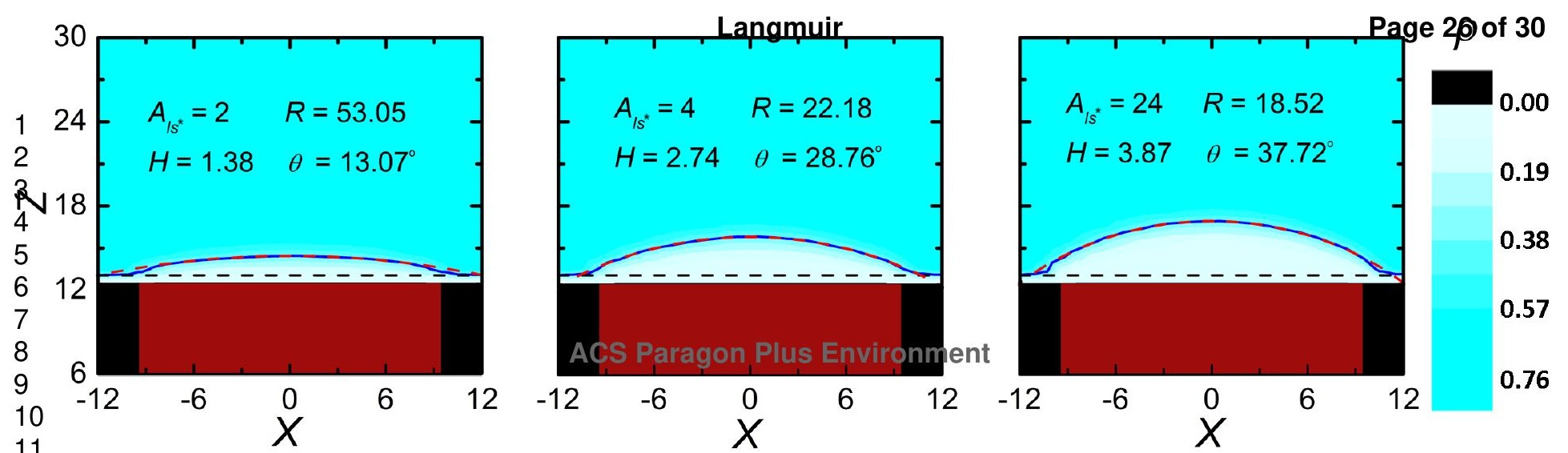




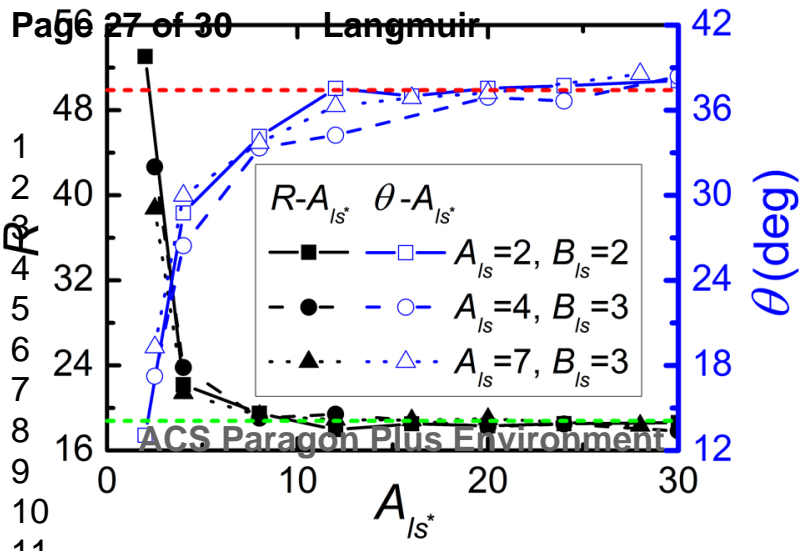




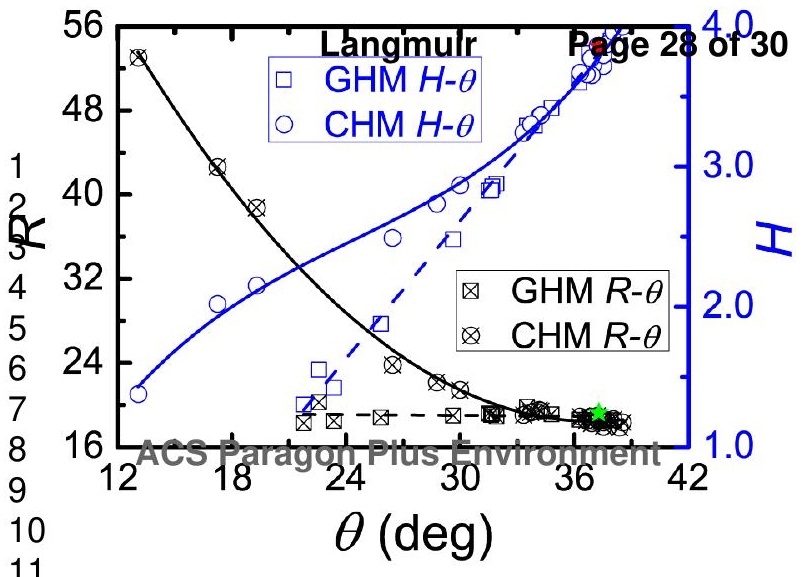


Page 29 of 30

(a)

Langmuir

(b)

1
2
3
4
5
6
7
8
9
10
11
12
13
14
15
16
17
18
19
20
21
22
23
24
25
26
27
28
29
30
31
32
33
34
35
36
38
39
40

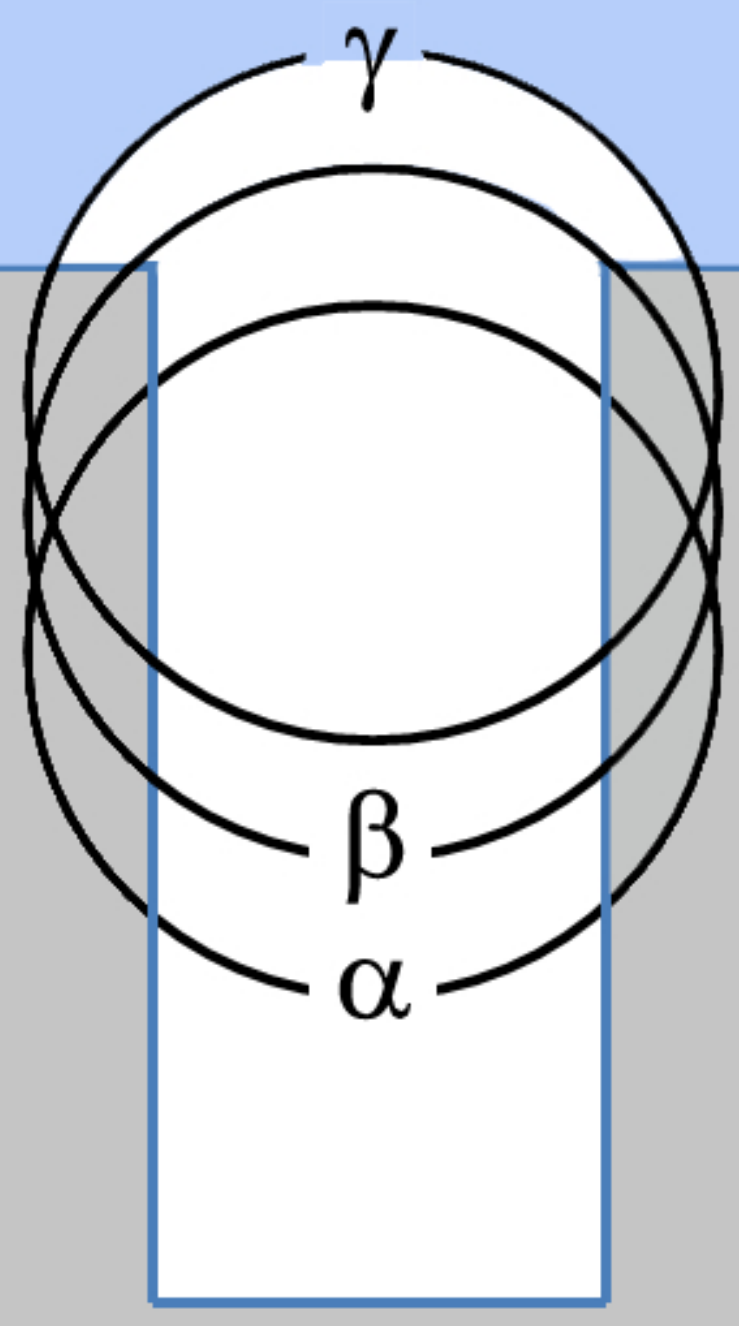

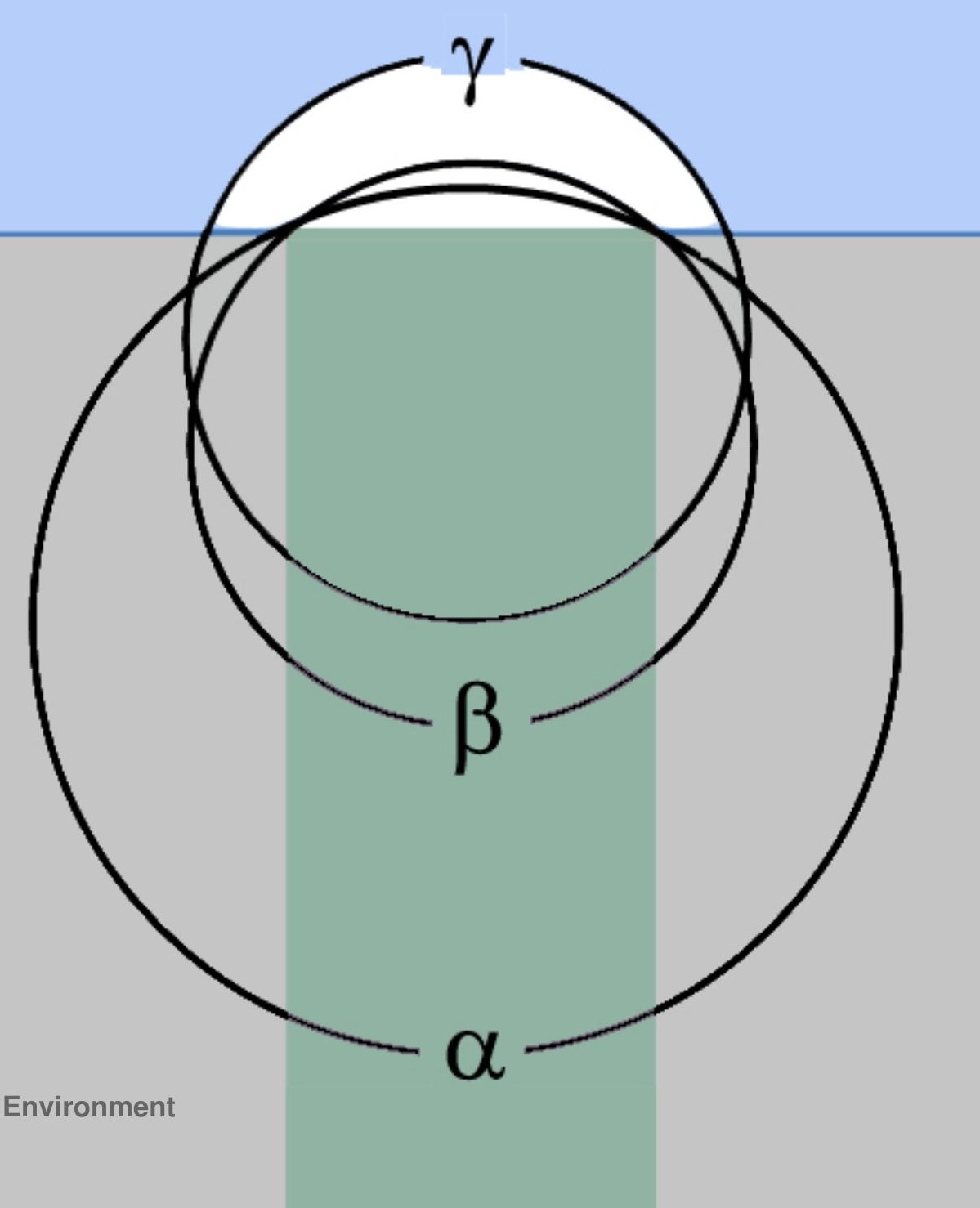




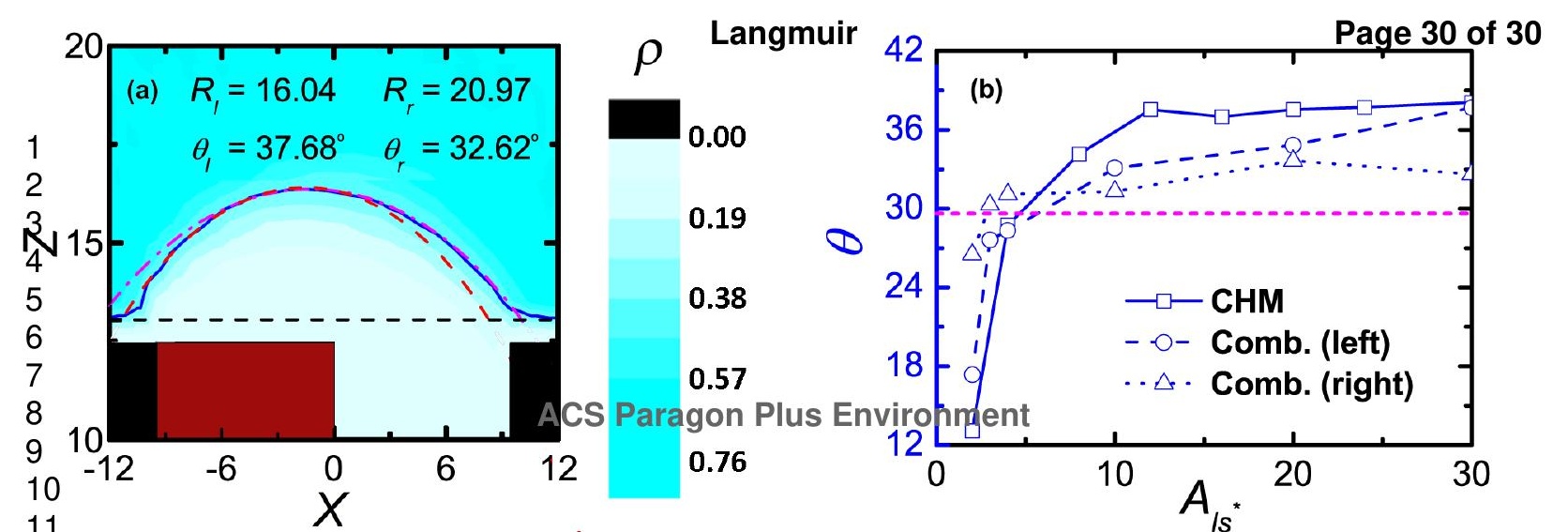

